\title{
PAPERS
}

\section{Preservation of postural control of transient lower oesophageal sphincter relaxations in patients with reflux oesophagitis}

\author{
A C Ireland, J Dent, R H Holloway
}

\begin{abstract}
Introduction-In normal subjects, transient lower oesophageal sphincter relaxations (TLOSRs) and gas reflux during belching are suppressed in the supine position. Supine reflux, however, is a feature of reflux disease.

Aims-To investigate whether postural suppression of TLOSRs and gas reflux is impaired in patients with reflux disease.

Patients-Ten patients with erosive oesophagitis.

Methods-Oesophageal manometry was performed during gastric distension with $750 \mathrm{ml}$ carbon dioxide. Measurements were made for 10 minutes before and after distension in both sitting and supine positions.

Results-In the sitting position gastric distension substantially increased the rate of gas reflux (median (interquartile range)), as evidenced by increases in oesophageal common cavities from 1 $(0-1) / 10 \mathrm{~min}$ to $7(5-10) / 10 \mathrm{~min}$ and TLOSRs from $1(1-1.5) / 10 \mathrm{~min}$ to 6 (2.5-8)/10 min. However, this effect was suppressed in the supine position in all but one patient (TLOSRs $0(0) / 10 \mathrm{~min}$ to 1 $(0-4.5) / 10 \mathrm{~min}$, common cavities $0(0) / 10$ min to $0.5(0-2) / 10 \mathrm{~min})$.

Conclusions-Postural suppression of TLOSRs and gas reflux is generally preserved in reflux disease.

(Gut 1999;44:313-316)
\end{abstract}

Department of Gastrointestinal Medicine, Royal Adelaide Hospital, Adelaide, SA 5000, Australia

A C Ireland

J Dent

R H Holloway

Correspondence to: Dr R Holloway, Department of Gastrointestinal Medicine, Royal Adelaide Hospital,

North Terrace, Adelaide, SA 5000, Australia.

Keywords: gastro-oesophageal reflux; lower oesophageal sphincter; posture; oesophageal manometry

In normal subjects and most patients with gastro-oesophageal reflux disease, reflux of acid occurs predominantly during transient lower oesophageal sphincter (LOS) relaxations (TLOSRs). ${ }^{1-4}$ These relaxations are also the major mechanism underlying the reflux of gas during belching. ${ }^{56}$ We have proposed therefore that acid reflux may be a variant of the belch reflex. ${ }^{5}$

In normal subjects, TLOSRs and gas reflux are almost completely suppressed in the supine posture. ${ }^{5}$ Reflux disease is characterised by an increased frequency of reflux episodes and, particularly in patients with more severe oesophagitis, by increased levels of recumbent reflux. ${ }^{7-13}$ These observations suggest that there may be defective postural suppression of TLOSRs in patients with reflux disease. The aim of this study therefore was to investigate the influence of posture on TLOSRs and gas reflux in patients with erosive or ulcerative reflux oesophagitis.

\section{Methods}

PATIENTS

We studied 10 patients, all men, with gastrooesophageal reflux disease. Their mean age was 59 (42-71) years and all had erosive oesophagitis (Hetzel grade 2-3). ${ }^{14}$ All patients gave written informed consent and the study was approved by the research ethics committee of the Royal Adelaide Hospital.

\section{MANOMETRIC TECHNIQUE}

Oesophageal manometry was performed using an eight channel assembly incorporating a sleeve sensor. Intragastric pressure was recorded by a side hole located $1 \mathrm{~cm}$ beyond the distal margin of the sleeve. Side holes at the proximal sleeve margin and $4,8,12$, and $16 \mathrm{~cm}$ more proximally recorded motility in the oesophageal body, and a side hole in the pharynx monitored swallowing. The sleeve and gastric and oesophageal side holes were perfused with degassed distilled water at a rate of 0.6 $\mathrm{ml} / \mathrm{min}$ using a low compliance pneumohydraulic capillary infusion system. The pharyngeal side hole was perfused at a rate of 0.3 $\mathrm{ml} / \mathrm{min}$ to obtain an accurate indication of the occurrence of swallowing but minimise the effect of the assembly on the swallow rate. Each lumen was connected to external pressure transducers (Deseret Medical Inc, Sandy, Utah, USA), the output of which was displayed on a 12 channel polygraph (model 7D; Grass Instruments, Quincy, Massachusetts, USA) at a paper speed of $2.5 \mathrm{~mm} / \mathrm{s}$.

Abbreviations used in this paper: LOS, lower oesophageal sphincter; TLOSR, transient LOS relaxation. 


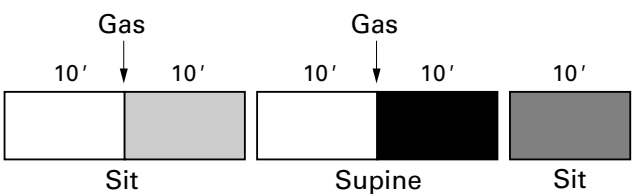

Figure 1 Schematic representation of the protocol for posture and gas loading followed in the study.

PROTOCOL

Studies were performed after a four hour fast and, in smokers, after at least six hours abstinence from smoking. Drugs that might affect oesophageal motility were discontinued for 24 hours before the study. After topical nasal anaesthesia, the catheter was introduced and positioned with the sleeve straddling the
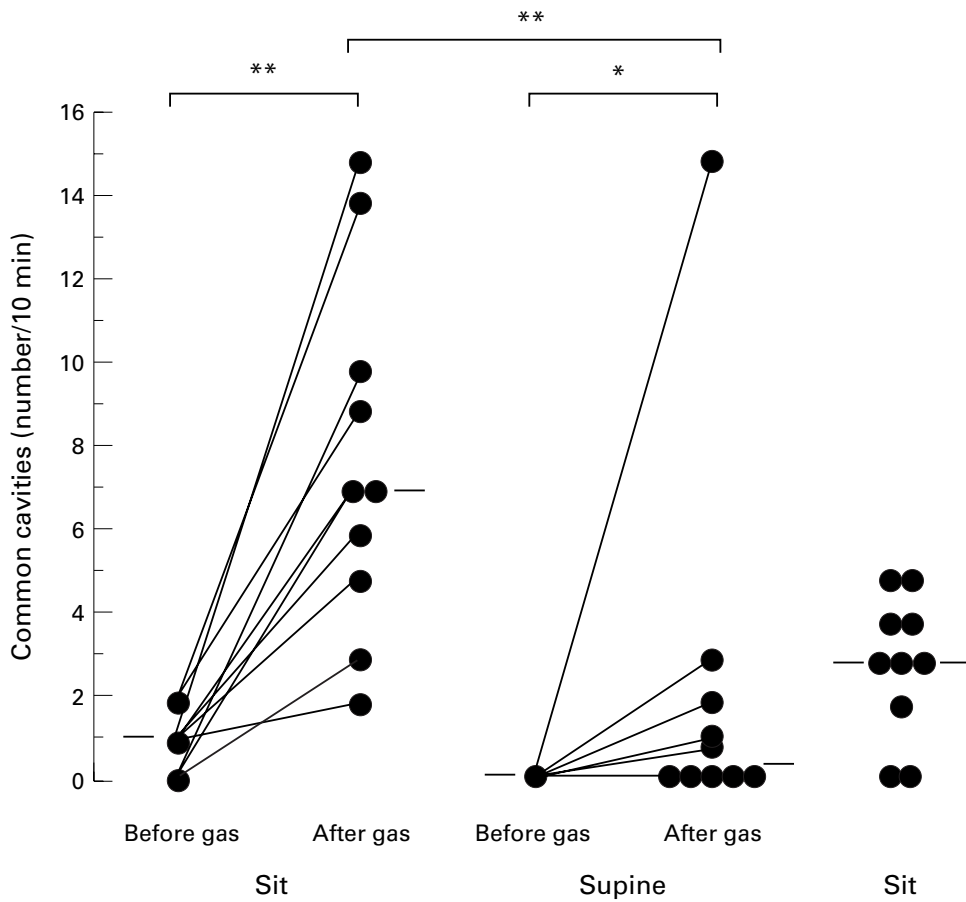

Figure 2 Rate of oesophageal common cavities before and after gastric distension with gas. Each dot repesents data for an individual patient. The horizontal bars indicate median values. ${ }^{\star} p<0.05,{ }^{\star *} p<0.01$.
Sitting

(116 episodes)

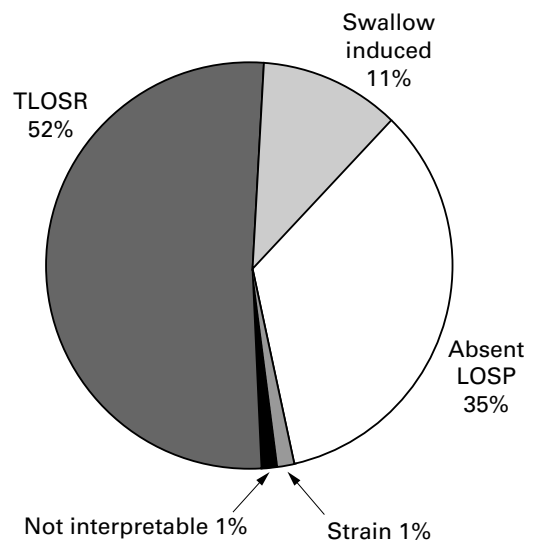

Supine (22 episodes)

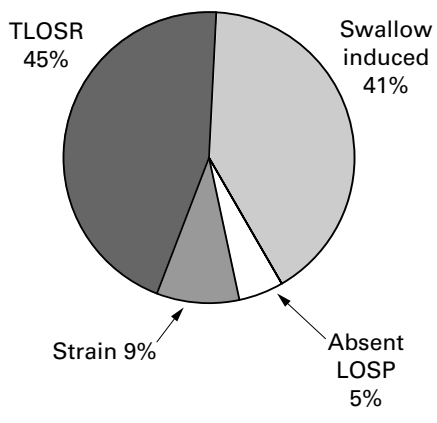

Figure 3 Effect of posture on patterns of lower oesophageal sphincter (LOS) and oesophageal motility associated with oesophageal common cavities. $p<0.01$ supine $v$ sitting. TLOSR, transient LOS relaxation; LOSP, LOS pressure; swallow induced, swallow induced LOS relaxation.
LOS. The patients were then allowed to accommodate to the assembly for 15 minutes.

Figure 1 is a schematic representation of the protocol. With the patients sitting, baseline recordings were made for 10 minutes, after which the stomach was distended abruptly with $750 \mathrm{ml} \mathrm{CO}_{2}$ by the instillation of a two part radiological mixture (Fields Negative C; Fields Group Chemicals, Sydney, NSW, Australia) into the stomach via the large central channel of the assembly. ${ }^{56}$ Recordings were continued for 10 minutes. The patients then lay supine and recordings were made for 10 minutes, after which the stomach was again distended with $750 \mathrm{ml} \mathrm{CO}$ and recordings continued for a further 10 minutes. Immediately after the second post-gas period, the patients sat up and another 10 minutes of recordings were made. During the study, subjects were instructed not to belch.

\section{DATA ANALYSIS}

Basal LOS pressure was measured at end expiration and referenced to intragastric pressure. A visual mean was taken for each minute of the 10 minute recording period and an overall mean calculated. The tracings were analysed for the presence of common cavities and the occurrence of TLOSRs. TLOSRs were scored according to previously defined criteria. ${ }^{15}$ Oesophageal common cavities were used as a marker of gas reflux, and defined as an abrupt increase in intraoesophageal pressure to intragastric pressure in at least the two distal oesophageal recording channels. ${ }^{56}$

\section{STATISTICAL ANALYSIS}

The frequencies of TLOSRs and oesophageal common cavities were analysed using the Friedman and Wilcoxon signed rank tests. Basal LOS pressure was analysed using analysis of variance and the paired Student's $t$ test. Data for TLOSRs and common cavities are expressed as median (interquartile range); data for basal LOS pressure are expressed as mean (SEM). p $<0.05$ was accepted as indicating statistical significance.

\section{Results}

When in the sitting position, patients exhibited a low rate of common cavities. Gastric distension produced a prompt and sevenfold increase in the rate of oesophageal common cavities $(p<0.01$; fig 2$)$. When the patients lay supine, the rate of common cavities fell to predistension levels and subsequent gastric distension induced a small but significant increase in the rate of common cavities $(\mathrm{p}<0.05)$. The rate of common cavities in the 10 minutes after distension when the patients were supine was lower in nine of the 10 patients than that when they were sitting. The median was significantly lower as a group $(0.5(0-2) / 10 \mathrm{~min} ; \mathrm{p}<0.01)$ when supine than when sitting $(7(5-10) / 10$ min). One patient, however, retained a high rate of gas reflux in the supine position. On resuming the sitting position, there was no significant increase in the rate of common cavities (3 (2-4)/10 min). 


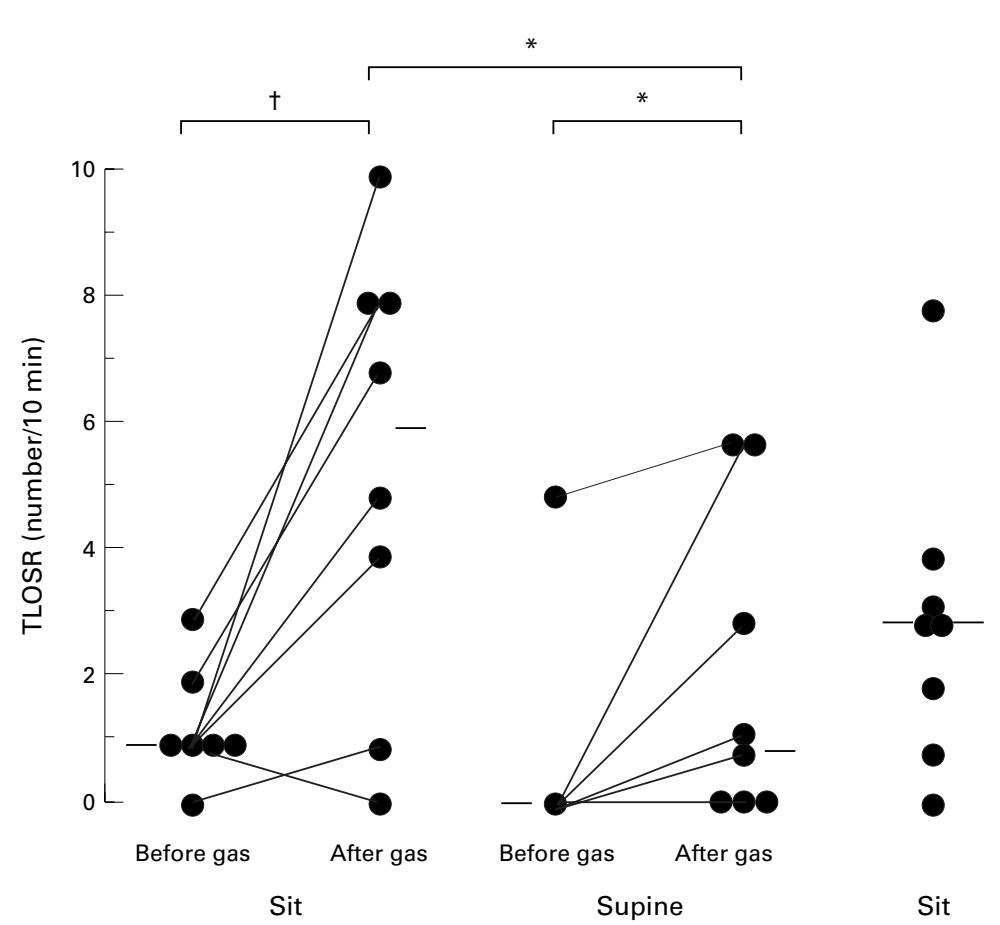

Figure 4 Rate of transient lower oesophageal sphincter relaxations (TLOSRs) before and after gastric distension with gas. Each dot repesents data for an individual patient. The horizontal bars indicate median values. ${ }^{\star} p<0.05$, $t p<0.03$. Basal LOS pressure was similar in the sitting and supine positions and was not affected by gastric distension in either position (fig 5).

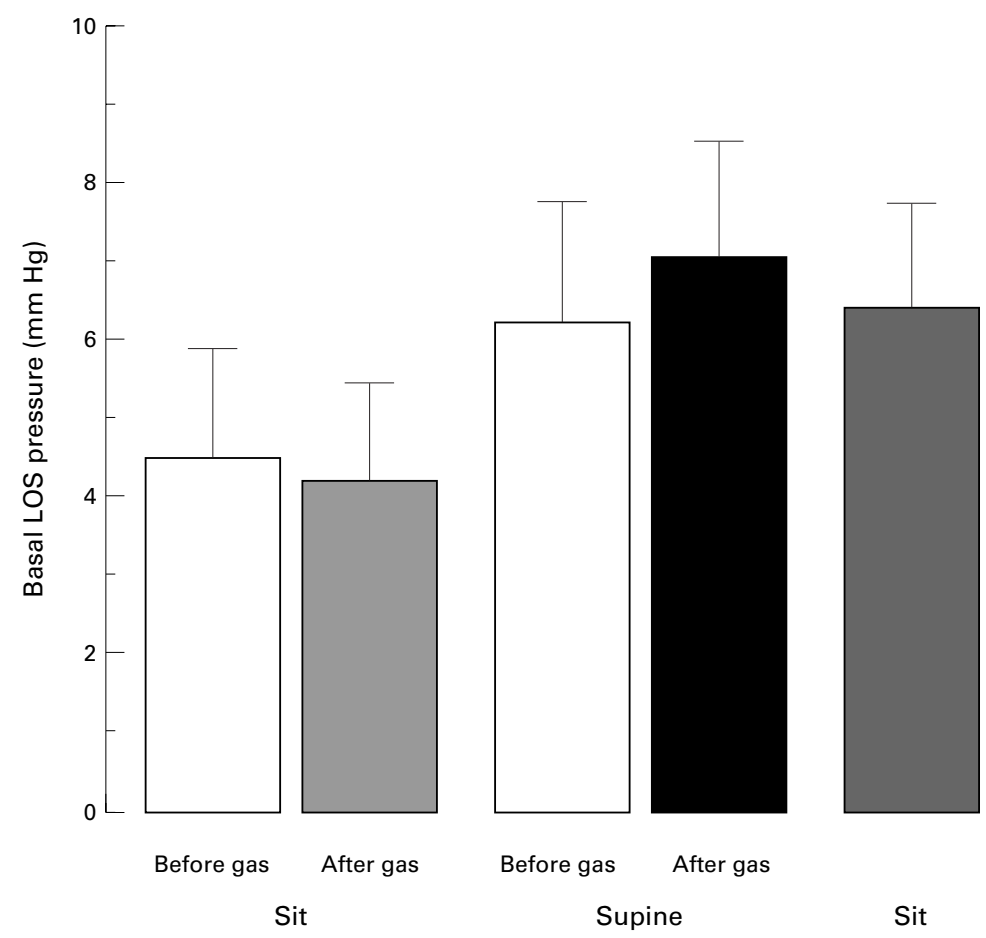

Figure 5 Effect of posture and gastric disension on basal lower oesophageal sphincter (LOS) pressure. The bars represent mean (SEM).

Figure 3 summarises the patterns of LOS and oesophageal motility associated with gas reflux. A total of 138 common cavity episodes was scored, 116 while sitting and 22 while supine. About half $(51 \%)$ of common cavities occurred during TLOSRs which was the most common mechanism overall; $16 \%$ occurred during swallow induced TLOSRs and 30\% during periods of absent LOS pressure. Posture had a significant effect on the patterns of motor activity associated with common cavities $(p<0.01)$; absent LOS pressure was more common while sitting, and swallow induced LOS relaxation more common while supine. However, almost all (9/10) of the common cavities that occurred during swallow induced LOS relaxation while supine occurred in one patient who was also the patient with the highest rate of supine common cavities.

Data on the rate of TLOSRs were available from only eight of the 10 patients because basal LOS pressure was too low in two patients to allow identification of these events. Gastric distension increased significantly the rate of TLOSRs in the sitting position from 1 $(1-1.5) / 10 \mathrm{~min}$ to $6(2.5-8) / 10 \mathrm{~min}(\mathrm{p}<0.03)$, and caused a smaller but significant increase when supine $(0 \quad(0)$ to $1(0-4.5) / 10 \mathrm{~min}$; $\mathrm{p}<0.05$; fig 4 ). Two patients had rates of TLOSR while supine that approached those when sitting. On resumption of the sitting position, there was no change in the rate of TLOSRs (3 (1.5-3.5)/10 min) compared with that during the post-distension supine period.

\section{Discussion}

This study was designed to test the hypothesis that postural control of TLOSRs and gas reflux is defective in patients with reflux oesophagitis. Our findings do not support this, at least as a general rule. Although gastric distension did increase the rates of both these events when the patient was in the supine posture, the rates in this position were significantly lower than when the patient was sitting, findings consistent with significant postural control. Moreover, TLOSRs and gas reflux were almost completely suppressed in all but three patients, results similar to those in normal subjects. ${ }^{516}$ These findings suggest that postural suppression of TLOSRs and gas reflux is generally preserved in patients with reflux disease although it may be less potent than in normal subjects and may even be defective in a small subgroup of patients.

Although our data argue against the notion of defective postural control of TLOSRs, they are not necessarily inconsistent with the observation of increased supine reflux in reflux disease. So-called "supine" reflux is an imprecise term in that patients are not necessarily supine. A more appropriate term would be "recumbent" reflux because patients may spend as much of their recumbent period in the lateral recumbent or even the semi-prone position rather than strictly supine as in the present study. TLOSRs and acid reflux occur in the lateral recumbent position in both normal subjects $^{17} 18$ and in patients with reflux disease, ${ }^{12} 19$ and in this position rates of TLOSRs are higher in patients with reflux disease than in normal subjects. ${ }^{20}{ }^{21}$ However, in patients with reflux disease, the rates of these events are significantly lower than in the sitting position, ${ }^{22}$ consistent with a degree of postural suppression in the lateral recumbent position also. 
Our previous studies in normal subjects have shown that suppression of TLOSRs in the supine posture is virtually complete in all normal subjects. ${ }^{5}{ }^{16}$ One of our patients, however, failed to show any suppression of TLOSRs or common cavities in the supine posture, and exhibited rates of these events that were substantially higher than that observed in our previous studies in normal subjects. Two other patients also had supine rates of TLOSRs that were higher than that in our previous controls, although, in both patients, their rate when supine was lower than that when sitting. These observations suggest that a subgroup of patients, for example those with predominant recumbent reflux, may have defective postural control of TLOSRs. Our patients were chosen on the basis of proven oesophagitis and not on the basis of supine reflux on 24 hour oesophageal $\mathrm{pH}$ monitoring. In patients with endoscopy-negative reflux disease or mild reflux oesophagitis, who constitute the majority of patients with reflux disease, the acid exposure is predominantly upright, diurnal, and postprandial. ${ }^{23}$ Nevertheless, the degree of recumbent acid exposure in such patients is higher than that in controls. ${ }^{9} 10121324$

It is also possible that supine reflux occurs predominantly through mechanisms other than TLOSRs that are not susceptible to postural suppression. Patients with reflux oesophagitis exhibit a heterogeneity of reflux mechanisms such that, although TLOSRs are the most common mechanism of reflux in most patients, another mechanism such as absent basal LOS pressure is the most important mechanism in some patients. ${ }^{4}$ In the present study, a higher proportion of common cavities occurred during swallow induced LOS relaxation in the supine posture. This mechanism occurred almost entirely in the one patient with the highest rate of common cavities when supine. Interestingly, however, we observed a reduction in the occurrence of gas reflux during absent basal LOS pressure.

The mechanism of postural suppression of TLOSRs remains unclear. The concept that fluid bathing the gastric cardia triggers inhibitory reflexes ${ }^{5}{ }^{25}$ has not been supported by subsequent studies in dogs, in which removal of the fluid did not remove the strong postural suppression. ${ }^{26}$ Likewise, the effects do not appear to be mediated through the vestibular system. ${ }^{16}$ It is possible that the supine posture imposes mechanical effects on the cardia that may influence patterns of LOS relaxation or the sensory mechanisms responsible for triggering TLOSRs. ${ }^{27}$ Postural suppression of reflux as the result of absent basal LOS tone may also be a reflection of mechanical effects on the LOS segment when supine.

In summary, we have shown preservation of postural suppression of TLOSRs and gas reflux in reflux disease. The mechanisms underlying this postural suppression, however, remain to be determined.
This study was supported by a project grant from the NHMRC A C I was supported by a travelling fellowship from the Roya College of Physicians in Edinburgh. The technical assistance of Marcus Tippett is gratefully acknowledged.

1 Dent J, Holloway RH, Toouli J, et al. Mechanisms of lower oesophageal sphincter incompetence in patients with symptomatic gastroesophageal reflux. Gut 1988;29:1020-

2 Dodds WJ, Dent J, Hogan WJ, et al. Mechanisms of gastroesophageal reflux in patients with reflux esophagitis. $N$ Engl f Med 1982;307:1547-52.

3 Schoeman MN, Tippett MD, Akkermans LM, et al. Mechanisms of gastroesophageal reflux in ambulant healthy human subjects. Gastroenterology 1995;108:83-91.

4 Penagini R, Schoeman MN, Dent J, et al. Motor events underlying gastro-oesophageal reflux in ambulant patients with reflux oesophagitis. Fournal of Neurogastroenterology with reflux oesophagitis. Four

and Motility 1996;8:131-41.
5 Wyman JB, Dent J, Heddle R, et al. Control of belching by the lower oesophageal sphincter. Gut 1990;31:639-46.

6 Holloway R, Wyman J, Dent J. Failure of transient lower oesphageal sphincter relaxation in response to gastric distention in patients with achalasia: evidence for neural mediation of transient lower oesphageal sphincter relaxations. Gut 1989;30:762-7.

7 de Caestecker JS, Blackwell JN, Pryde A, et al. Daytime gastro-oesophageal reflux is important in oesophagitis. Gut 1987;28:519-29.

8 Robertson D, Aldersley M, Shepherd H, et al. Patterns of acid reflux in complicated oesophagitis. Gut 1987;28: 1484-8.

9 Schindlbeck NE, Heinrich C, Konig A, et al. Optimal thresholds, sensitivity, and specificity of long-term $\mathrm{pH}$ metry for the detection of gastroesophageal reflux disease. Gastroenterology 1987;93:85-90.

10 Rokkas T, Sladen GE. Ambulatory esophageal pH recording in gastroesophageal reflux: relevance to the developing in gastroesophageal reflux: relevance to the develop-
ment of esophagitis. Am 7 Gastroenterol 1988;83:629-32.

11 Jenkinson LR, Norris TL, Barlow AP, et al. Acid reflux and oesophagitis-day or night? Gullet 1990;1:36-44

12 Masclee A, De Best A, De Graaf R, et al. Ambulatory 24 hour $\mathrm{pH}$-metry in the diagnosis of gastroesophageal reflux disease. Determination of criteria and relation to endoscopy. Scand F Gastroenterol 1990;25:225-30.

13 Ghillebert G, Demeyere AM, Janssens J, et al. How well can quantitative 24-hour intraesophageal $\mathrm{pH}$ monitoring distinguish various degrees of reflux disease? Dig Dis Sci 1995; 40:1317-24.

14 Hetzel DJ, Dent J, Reed WD, et al. Healing and relapse of severe peptic esophagitis after treatment with omeprazole. Gastroenterology 1988;95:903-12.

15 Holloway RH, Penagini R, Ireland AC. Criteria for objective definition of transient lower esophageal sphincter relaxation. Am f Physiol 1995;268:G128-33.

16 Ireland A, Dent J, Holloway RH. The role of head position in the postural control of transient lower oesophagea sphincter relaxations and belching. Gullet 1992;2:81-4.

17 Dent J, Dodds WJ, Friedman RH, et al. Mechanism of gastroesophagal reflux in recumbent asymptomatic subjects. $\mathcal{F}$ Clin Invest 1980;65:256-67.

18 Mittal RK, McCallum RW. Characteristics of transient lower esophageal sphincter relaxation in humans. Am $\mathcal{f}$ Physiol 1987;252:G636-41.

19 Mittal RK, McCallum RW. Characteristics and frequency of transient relaxations of the lower esophageal sphincter on patients with reflux esophagitis. Gastroenterology 1988;95: 593-9.

20 Holloway RH, Kocyan P, Dent J. Provocation of transient lower esophageal sphincter relaxations by meals in patients with symptomatic gastroesophageal reflux. Dig Dis Sci 1991;36:1034-9.

21 Penagini R, Bianchi PA. Effect of morphine on gastroesophageal reflux and transient lower esophageal sphincter relaxation. Gastroenterology 1997;113:409-14

22 Freidin N, Mittal RK, McCallum RW. Does body posture affect the incidence and mechanism of gastro-oesophageal reflux? Gut 1991;32:133-6.

23 Gudmundsson K, Johnsson F, Joelsson B. The time pattern of gastroesophageal reflux. Scand F Gastroenterol 1988;23: $75-9$.

24 Orr WC, Allen ML, Robinson M. The pattern of nocturnal and diurnal esophageal acid exposure in the pathogenesis of erosive mucosal damage. Am 7 Gastroenterol 1994;89: 509-12.

25 Dougherty RW. Esophageal innervation and the eructation reflex in sheep. Am F Vet Res 1958;19:115-28.

26 Little AF, Cox MR, Martin CJ, et al. Influence of posture on transient lower oesophageal sphincter relaxation and gastro-oesophageal reflux in the dog. $\mathcal{F}$ Gastroenterol Hepatol 1989;4:49-54.

27 Franzi SJ, Martin CJ, Cox MR, et al. Response of canine lower esophageal sphincter to gastric distension. $A m \mathcal{F}$ Physiol 1990;259:G380-5. 